\title{
QUELQUES REMARQUES SUR LES RELATIONS ENTRE LES NOTIONS D'ECART REGULIER ET DE DISTANCE
}

\author{
N. ARONSZAJN
}

Madame A. Frink a donné récemment* une nouvelle démonstration très élégante du théorème suivant de M. E. W. Chittenden: $S i$ dans un espace E la notion de limite est définie par un écart régulier $r, \dagger$ elle peut être définie également par une distance $\rho$.

La démonstration de Mme Frink repose sur une construction de la distance $\rho$ qui, à cause de sa simplicité, permet d'établir des relations étroites entre les propriétés métriques de l'écart $r$ et de la distance $\rho$.

Dans ce qui suit, nous allons indiquer des relations de ce genre dans quelques cas simples. Par $r$ nous désignerons un écart régulier défini dans l'espace $E$ et par $\rho$ la distance correspondante construite par Mme Frink.

1. Les écarts complets. Nous dirons qu'une suite de points $\left\{p_{n}\right\}$ de $E$ est une suite de Cauchy selon $r$, si $\lim _{i=\infty, j=\infty} r\left(p_{i}, p_{j}\right)=0$. Si toute suite de Cauchy selon $r$ converge dans $E$, l'écart $r$ sera dit complet.

De la démonstration de Mme. Frink résulte qu'il existe deux fonctions croissantes $\mu_{1}(\eta)$ et $\mu_{2}(\eta)$ telles que $\mu_{i}(\eta)>0$ pour $\eta>0, \mu_{i}(0)$ $=\lim _{\eta=0} \mu_{i}(\eta)=0$, et que

$$
\mu_{1}(r(p, q)) \leqq \rho(p, q) \leqq \mu_{2}(r(p, q)),
$$

pour tous les $p, q$ de $E$.

Il s'ensuit de (1) immédiatement qu'une suite de Cauchy selon $r$ est en même temps une suite de Cauchy selon la distance $\rho$ et vice versa. De ce fait découle le théorème suivant:

ThÉor Ème 1. Pour que la distance $\rho$ soit complète, il faut et il suffit que l'écart r le soit.

2. La presque-convexité. Un écart $r^{\prime}$ est dit presque-convexe, si, pour tous $a, b$ de $E$ et tout $\eta>0$, il existe un $c$ de $E$ tel que

* Ce Bulletin, vol. 43 (1937), p. 133.

$\dagger$ On dit d'apres $M$. Fréchet que dans $E$ est défini un écart régulier $r$, si pour tout couple de points $(p, q)$ de $E$ est défini un nombre $r(p, q) \geqq 0$ tel que: $(1) r(p, q)=0$ équivaut a $p=q$, (2) $r(p, q)=r(q, p)$, et (3) il existe une fonction positive croissante $\delta(\epsilon)$, pour $\epsilon>0$, telle que, si $r(x, y) \leqq \delta(\epsilon)$ et $r(y, z) \leqq \delta(\epsilon), r(x, z) \leqq \epsilon$.

$\ddagger$ Les fonctions $\mu_{i}(\eta)$ ne dépendent que de la fonction $\delta(\epsilon)$ qui correspond à l'écart $r$. Pour $\delta(\epsilon)=\epsilon / 2$, on peut prendre $\mu_{1}(\eta)=\eta / 4$ et $\mu_{2}(\eta)=\eta$. 


$$
\left|r^{\prime}(a, c)-\frac{1}{2} r^{\prime}(a, b)\right|<\eta,\left|r^{\prime}(c, b)-\frac{1}{2} r^{\prime}(a, b)\right|<\eta .^{*}
$$

Cette propriété entraîne évidemment la suivante:

(A) Pour tous $a, b$ de $E, \eta>0$, et $n$ entier positif, il existe une suite de points $c_{k},\left(k=1,2, \cdots,\left(2^{n}-1\right)\right)$, tels que, en posant $a=c_{0}$ et $b=c_{2^{n}}$, on ait

$$
\left|r^{\prime}\left(c_{k}, c_{k+1}\right)-\frac{1}{2^{n}} r^{\prime}(a, b)\right|<\eta, \quad k=0,1,2, \cdots,\left(2^{n}-1\right) .
$$

Supposons maintenant que l'écart $r$ satisfait à la condition suivante:

(X) Sir $r(x, y) \leqq \epsilon$ et $r(y, z) \leqq \epsilon, r(x, z) \leqq 2 \epsilon . \dagger$

THÉOR غ̀me 2. Si l'écart $r$, satisfaisant à (X), est presque-convexe, la distance $\rho$ l'est également.

Démonstration. Revenons à la définition de la distance $\rho$. Pour un écart $r$ satisfaisant à $(\mathrm{X})$, elle s'écrit $f$

$$
\rho(a, b)=\text { borne inf. } \sum_{i=0}^{N-1} r\left(p_{i}, p_{i+1}\right),
$$

$\left\{p_{i}\right\},(i=0,1, \cdots, N)$, parcourant toutes les suites finies de points de $E$ avec $p_{0}=a$ et $p_{N}=b$.

Pour un $\eta>0$ quelconque choisissons une telle suite avec

$$
\left|\rho(a, b)-\sum_{i=0}^{N-1} r\left(p_{i}, p_{i+1}\right)\right|<\frac{\eta}{4} .
$$

Dans la suite $\left\{p_{i}\right\}$ soit $p_{k}$ le dernier terme pour lequel la somme $\sum_{i=0}^{k-1} r\left(p_{i}, p_{i+1}\right)$ est encore $\leqq \frac{1}{2} \rho(a, b)$. D'après (2. 2$), k<N$. On a donc

$$
0 \leqq \xi<r\left(p_{k}, p_{k+1}\right), \quad \text { où } \quad \xi=\frac{1}{2} \rho(a, b)-\sum_{i=0}^{k-1} r\left(p_{i}, p_{i+1}\right) .
$$

* La notion de presque-convexité a un sens pour les écarts les plus généra ux, mais elle présente le plus d'intérêt quand $r^{\prime}$ est une distance. Elle a été introduite pour les distances dans notre thèse Sur la métrique et la métrisation, Varsovie, 1930 (non publiée). M. K. Menger a mentionné cette notion dans son article Bericht ïber metrische Geometrie, Jahresbericht der deutschen Mathematiker-Vereinigung, vol. 40 (1931), p. 207. Il est à remarquer que pour une distance $r^{\prime}$, la presque-convexité entraîne la propriété plus forte suivante: pour toute décomposition $r^{\prime}(a, b)=\alpha+\beta$, avec $\alpha \geqq 0, \beta \geqq 0$, et pour tout $\eta>0$, il existe un point $c$ avec $\left|r^{\prime}(a, c)-\alpha\right|<\eta$ et $\left|r^{\prime}(c, b)-\beta\right|<\eta$.

$\dagger$ La condition $(\mathrm{X})$ signifie que $\delta(\epsilon)=\epsilon / 2$.

$\ddagger$ Cf. A. Frink, loc. cit., p. 135. 
Choisissons alors un $n$ entier positif de sorte que

$$
\frac{1}{2^{n}} r\left(p_{k}, p_{k+1}\right)<\frac{\eta}{4}
$$

et considérons la suite $\left\{q_{j}\right\}$, existant d'après $(\mathrm{A})$, telle que

$$
\begin{aligned}
& q_{0}=p_{k}, q_{2^{n}}=p_{k+1},\left|r\left(q_{j}, q_{j+1}\right)-\frac{1}{2^{n}} r\left(p_{k}, p_{k+1}\right)\right|<\frac{\eta}{4 \cdot 2^{n}}, \\
& j=0,1, \cdots,\left(2^{n}-1\right) .
\end{aligned}
$$
donc

Dans la suite $\left\{q_{j}\right\}$ soit $q_{h}$ le dernier terme avec $\sum_{j=0}^{h-1} r\left(q_{j}, q_{j+1}\right) \leqq \xi$,

$$
0 \leqq \xi-\sum_{j=0}^{h-1} r\left(q_{j}, q_{j+1}\right)<r\left(q_{h}, q_{h+1}\right)<\frac{\eta}{4}+\frac{\eta}{4 \cdot 2^{n}}<\frac{2 \eta}{4} \cdot *
$$

D'après la définition de la distance $\rho$, on tire des equations (2.4) et (2.7)

$$
\begin{aligned}
\rho\left(a, q_{h}\right) & \leqq \rho\left(a, p_{k}\right)+\rho\left(p_{k}, q_{h}\right) \\
& \leqq \sum_{i=0}^{k-1} r\left(p_{i}, p_{i+1}\right)+\sum_{j=0}^{h-1} r\left(q_{j}, q_{j+1}\right) \leqq \frac{1}{2} \rho(a, b) .
\end{aligned}
$$

Les inégalités de (2.3) à (2.7) nous donnent ensuite

$$
\begin{aligned}
\rho\left(q_{h}, b\right) \leqq & \rho\left(q_{h}, p_{k+1}\right)+\rho\left(p_{k+1}, b\right) \\
\leqq & \sum_{j=h}^{2^{n}-1} r\left(q_{j}, q_{j+1}\right)+\sum_{i=k+1}^{N-1} r\left(p_{i}, p_{i+1}\right) \\
= & {\left[\sum_{j=0}^{2^{n-1}} r\left(q_{j}, q_{j+1}\right)-\sum_{j=0}^{h-1} r\left(q_{j}, q_{j+1}\right)\right] } \\
& \quad+\left[\sum_{i=0}^{N-1} r\left(p_{i}, p_{i+1}\right)-\sum_{i=0}^{k} r\left(p_{i}, p_{i+1}\right)\right] \\
< & r\left(p_{k}, p_{k+1}\right)+\frac{\eta}{4}-\xi+\frac{2 \eta}{4} \\
& +\rho(a, b)+\frac{\eta}{4}+\xi-\frac{1}{2} \rho(a, b)-r\left(p_{k}, p_{k+1}\right) \\
= & \frac{1}{2} \rho(a, b)+\eta .
\end{aligned}
$$

* $\mathrm{Si} h=2^{n}$, les inégalités (2.7) restent vraies à condition qu'on y efface le membre $r\left(q_{h}, q_{h+1}\right)$ (ceci se déduit de (2.4) et (2.6). 
En posant $c=q_{h}$ on déduit des inégalités (2.8) et (2.9) immédiatement que

$$
\left|\rho(a, c)-\frac{1}{2} \rho(a, b)\right|<\eta \quad \text { et }\left|\rho(c, b)-\frac{1}{2} \rho(a, b)\right|<\eta,
$$

ce qu'il fallait démontrer.

3. Remarque sur la notion de distance géodésique. Le Théorème 2 peut être considéré comme une conséquence d'un théorème concernant la distance géodésique dans un espace métrique général. On peut définir la distance géodésique de différentes manières. * D'un certain point de vue il est avantageux de la définir de manière suivante:

Soit $r^{\prime}$ un écart soumis uniquement aux conditions suivantes:

$$
r^{\prime}(x, y) \geqq 0, \quad r^{\prime}(x, x)=0 .
$$

On appelle $\epsilon$-chaîne unissant $a$ et $b$ une suite $\left\{p_{i}\right\},(i=0,1, \cdots, N)$, telle que

$$
p_{0}=a, p_{N}=b, r^{\prime}\left(p_{i}, p_{i+1}\right) \leqq \epsilon, \quad i=0,1, \cdots, N-1 .
$$

On appelle longueur de la chaîne $\left\{p_{i}\right\}$, la quantité

$$
L\left\{p_{i}\right\}=\sum_{i=0}^{N-1} r^{\prime}\left(p_{i}, p_{i+1}\right) .
$$

Une chaîne $\left\{p_{i}^{\prime}\right\}$ est dite subdivision de $\left\{p_{i}\right\}$, si elle s'obtient de cette dernière en intercalant entre certains (ou entre tous) éléments consécutifs $p_{i}, p_{i+1}$ de nouveaux éléments.

Nous dirons qu'une suite des chaînes $\left\{\left\{p_{i}{ }^{(k)}\right\}\right\},(k=1,2, \cdots)$, est une suite convergente de subdivisions liant a à $b$, si (1) $\left\{p_{i}{ }^{(k)}\right\}$ sont des $\boldsymbol{\epsilon}_{k}$-chaînes unissant $a$ et $b$, avec $\lim _{k=\infty} \epsilon_{k}=0 ;(2)\left\{p_{i}{ }^{(k+1)}\right\}$ est une subdivision de $\left\{p_{i}{ }^{(k)}\right\}$, et (3) $\lim _{k=\infty} L\left\{p_{i}{ }^{(k)}\right\}$ existe (mais peut être fini ou infini).

La "distance" géodésique $\bar{\rho}(a, b)$ peut être maintenant définie comme suit:

$$
\bar{\rho}(a, b)=\text { borne inf. } \lim _{k=\infty} L\left\{p_{i}^{(k)}\right\}
$$

les $\left\{\left\{p_{i}{ }^{(k)}\right\}\right\}$ parcourant toutes les suites convergentes de subdivisions liant $a$ à $b . \dagger$

* M. K. Menger a considéré, dans les espaces distancés, une distance géodésique égale, d'après le modèle classique, à la borne inférieure des longueurs des arcs unissant deux points $a$ et $b$ de $E$ (cf. Mathematische Annalen, vol. 103 (1930), p. 492).

$\dagger$ Il est à remarquer que si $r^{\prime}$ est une distance complète, $\bar{\rho}$ se confond avec la distance géodésique formée d'après le modèle classique. 
On démontre pour $\bar{\rho}$ les propriétés suivantes: (i) $\bar{\rho}(x, y) \geqq 0$, $\bar{\rho}(x, x)=0$ (mais $\bar{\rho}(x, y)$ peut être infini, $\bar{\rho}(x, y)$ peut être $=0$ sans que $x=y$ et il se peut que $\bar{\rho}(x, y) \neq \bar{\rho}(y, x))$; (ii) $\bar{\rho}(x, y)+\bar{\rho}(y, z) \geqq \bar{\rho}(x, z)$. A cause de l'inégalité triangulaire (ii) on peut considérer $\bar{\rho}$ comme une sorte de distance.

On démontre de plus la propriété: (iii) la "distance" géodêsique $\bar{\rho}$, si elle est finie, est toujours presque-convexe.

En revenant maintenant aux conditions du Théorème 2, on prouve d'abord que la distance $\rho$ est égale à la distance géodésique construite à l'aide de l'écart $r$ et le théorème résulte alors de la propriété (iii).

4. Les écarts complets et presque-convexes. Les Théorèmes 1 et 2 donnent ensemble le théorème suivant:

THÉoR ̇̀me 3. Si l'écart $r$, satisfaisant à la condition (X) $d u$ \$2, est complet et presque-convexe, il en est de même de la distance $\rho$.

L'intérêt de ce théorème réside dans le fait qu'un espace avec une distance complète et presque-convexe possède des propriétés remarquables. Ainsi par exemple, dans un tel espace, deux points quelconques $a$ et $b$ peuvent être liés par un arc presque-géodésique de longueur inférieure à $\rho(a, b)+\epsilon$, pour $\epsilon>0$ quelconque. Il en résulte la propriété topologique suivante: l'espace est quasi-péanien.*

Paris, France

* D'après M. K. Borsuk on appelle ainsi un espace connexe, localement connexe, et métrisable à l'aide d'une distance complète. 